\section{Perceptual search time as a function of association value with practice effects controlled}

\author{
ELMER H. DAVIDSON* \\ University of North Carolina at Wilmington, Wilmington, N.C. 28401 \\ and \\ COLIN L. ROBERTSON \\ Oklahoma City University, Oklahoma, Okla. 73106
}

The effects of association value (AV) on perceptual search time was investigated in a design to control practice effects. Forty Ss were each exposed to four lists of trigrams (field) with instructions to find a particular one (target). No $S$ experienced more than one level of each factor of the 2 by 2 by 4 design. There were two AV levels of the target trigrams, two AV levels of the field list of trigrams, and four target positions. As expected, perceptual search time increased as the target was moved down the list. The analysis supported the hypothesis that AV reduced perceptual search time when the field and target have different AV. It was concluded that practice effects and perhaps S's failure to retain the targets of low AV during his search had influenced the results of previous studies.

While studying the effects of association value (AV) on the discriminability of a perceptual target, Portnoy, Portnoy, \& Salzinger (1964) demonstrated that when a target trigram of one AV was embedded in a field of trigrams of a different $\mathrm{AV}$, then perceptual search time was reduced to less than when the target and field were of the same AV. Their data also indicated that target trigrams of high $\mathrm{AV}$ were more rapidly detected than were those of low AV. No difference was indicated due to AV of the field list of trigrams.

Smith \& Egeth (1966) questioned the results of the above study on the grounds that the data did not permit an analysis of the search time per item as well as the possibility that Ss may have been using a search strategy of first letters rather than the complete trigram. Further, Smith and Egeth suggested that since the trigrams of the field were spread equally across a card, Ss may have wasted time in searching a previously searched area. They replicated the Portnoy, Portnoy, and Salzinger study with the target trigrams embedded in four positions of one vertical list of field trigrams. Their results did not support the hypothesis that AV affected perceptual search times.

Another study which dealt with the same phenomenon was that of Schulz \& Lovelace (1964). Their data indicated that when the target trigram was presented simultaneously with a horizontal field of eight trigrams, targets of low meaningfulness $(\mathrm{M})$ in low $\mathrm{M}$ fields were not detected as rapidly as were targets of high $\mathrm{M}$ in high $\mathrm{M}$ fields. They also

\footnotetext{
*The data for this report were collected while the senior author was on the faculty of Oklahoma City University.
}

presented data which indicated that practice had an effect on the perceptual search times. Smith \& Egeth (1966) indicated that the difference in search time for low and high $M$ situations in the Schulz \& Lovelace (1964) study was perhaps due to a different search strategy due to the horizontal placing of the trigrams which was similar to reading.

In a review of these studies, it seemed that Smith \& Egeth (1966) did improve upon the design of Portnoy, Portnoy, \& Salzinger (1964). Smith and Egeth seemed to control adequately for the per-item search time and for the possibly wasted time in repeating a previously searched area, but when they allowed each $S$ to do 16 searches, it became possible for Ss to learn that the target would appear in one of four areas and to concentrate their search there. Since Smith \& Egeth (1966) did not give the order in which the lists were presented to $\mathrm{Ss}$, perhaps a random order should be assumed; however, if the order was not random, this new search strategy may have been used even more extensively.

The study reported here was done as a replication of the three previous studies (Portnoy, Portnoy, \& Salzinger, 1964; Schulz \& Lovelace, 1964; Smith \& Egeth, 1966) in a design to control for possible additional search strategies produced by practice effects.

\section{SUBJECTS}

Forty undergraduate students of Oklahoma City University were used as Ss.

\section{DESIGN}

The stimulus conditions were kept similar to those used by Smith \& Egeth (1966) and Portnoy, Portnoy, \& Salzinger (1964). First, 49 trigrams of $0 \% \mathrm{AV}$ and 49 trigrams of $100 \% \mathrm{AV}$ were selected from the Glaze (1928) list to be used as field items. Also, 8 trigrams of $0 \%$ and 8 trigrams of $100 \%$ were selected to be used as targets. The first letter of the trigrams was balanced as well as possible from the Glaze (1928) list.

The trigrams were arranged in to 16 lists in order to form a 2 by 2 by 4 design. There were two levels $(0 \%$ and $100 \%)$ of target AV, two levels ( $0 \%$ and $100 \%)$ of . field $\mathrm{AV}$, and the target was embedded in one of four positions in each list. The positions were the 12 th, 23rd, 32nd, and 43rd, as used in the Smith \& Egeth (1966) study. Each list was presented on a single $81 / 2 \times 11$ in. sheet of paper, with the trigrams in two vertical columns separated as much as possible on the sheet. The 49 field items were ordered randomly, and the target was inserted at the appropriate position for each of the 16 lists. All items were typed in capitals and double spaced. Each $\mathrm{S}$ was presented with only four lists, and these were counterbalanced so that no $S$ experienced the same target-field relationship or the same position of the target more than once. The groups of four lists were assigned to $\mathrm{Ss}$ in block randomization so that each replication of the design was completed before another was started. The order of presentation of the four lists to $\mathrm{Ss}$ was determined randomly. Since no target-field relationship or position of target was repeated for any $S$, it appeared that practice effects would not occur, except possibly as a general set which would be balanced by the random presentation of the lists.

\section{PROCEDURE}

The Ss were run individually and were verbally instructed to always read down the columns, the left one first, and then the right one. They were instructed never to read from bottom to top or to skip across the page. They were further instructed to do the task as quickly as possible but without making a mistake.

Before the presentation of the list, $\mathrm{S}$ was shown the target on a white $3 \times 5$ in. card, which was exposed during the entire search time. Then $S$ turned over the list (face down before him) and started his search. Search time was the interval between turning over the list and S's verbal response when he pointed to the correct target. No errors were allowed and $S$ continued to search until the target was located. Search time was measured by use of a stopwatch, graduated in $1 / 5 \mathrm{sec}$.

\section{RESULTS}

The number of seconds required to locate the target trigram were used as scores for the data analysis. These data are summarized in Fig. 1. 


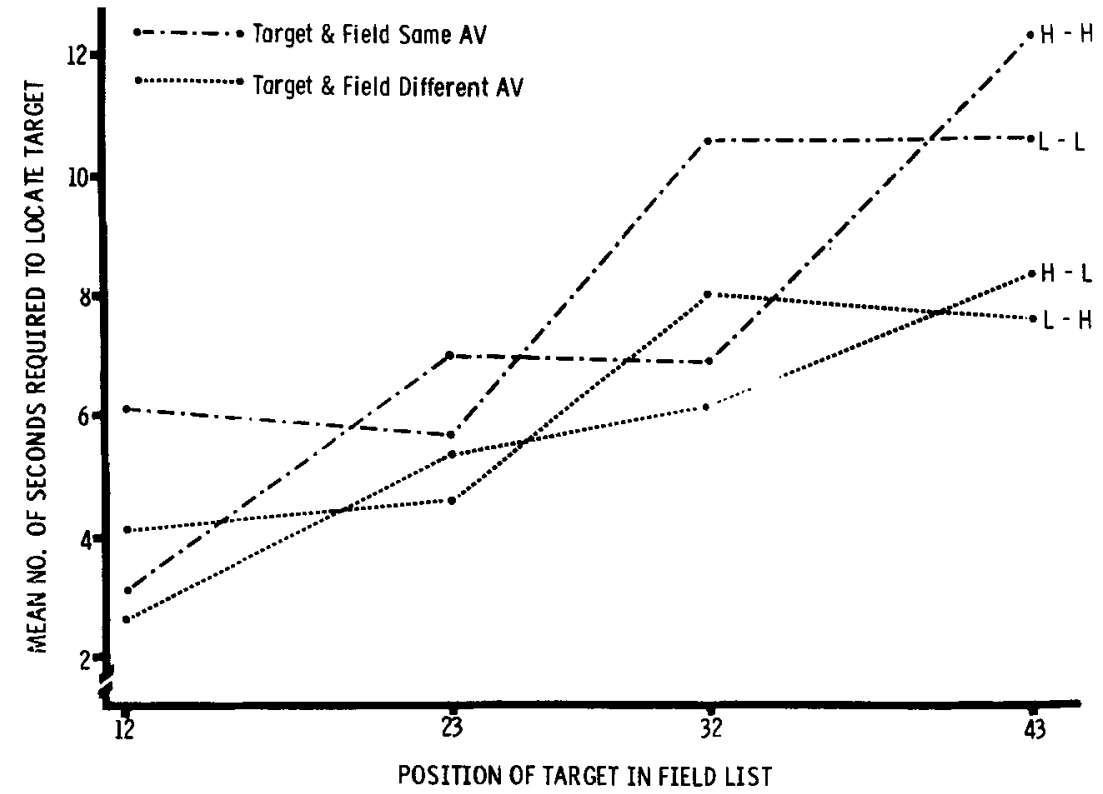

Fig. 1. Perceptual search time as a function of target and field $A V$ and position of target in the field list.

The position of the target trigram had the expected effect of requiring more time as the target was moved down the lists $[F(3,144)=7.24, p<.01]$. Neither the $\mathrm{AV}$ of the target nor the AV of the field produced a main effect; however, there was an interaction between those two variables $[F(1,144)=4.28, \quad p<.05]$ such that when the target was in a field of a different $A V$, search time was reduced below the search time that occurred when the target AV was the same as that of the field. None of the other interactions were statistically significant.

Since the design of the study depended upon the elimination of practice effects, the data were analyzed over the four trials that each $S$ experienced, and no differential practice effects were found.

$$
\text { DISCUSSION }
$$

As predicted, when practice effects were controlled, an interaction between $\mathrm{AV}$ of the target and AV of the field was produced. There is evidence that this interaction is not due to first-letter search, as Smith \& Egeth (1966) suggest. In the two conditions where search time was shorter ( $\mathrm{H}-\mathrm{L}$ and $\mathrm{L}-\mathrm{H})$, there were a total of 18 replications of the first letter of the target as a first letter of a field item. In the two conditions where search time was longer $(\mathrm{H}-\mathrm{H}$ and $\mathrm{L}-\mathrm{L})$, the first letter of the target was replicated as first letters of field items only eight times. If a first-letter strategy of search had been used, the search times of the conditions should have been reversed.

In spite of the foregoing supporting statements, during the data analysis, the Es began to wonder if short-term memory and interference had a role in producing the interaction found.

In the Portnoy, Portnoy, \& Salzinger (1964) study, it was indicated that the target of low AV was less readily detected. Since the trigrams of low AV (or M) were probably retained to a lesser degree after initial exposure (Underwood, 1964), the effect attributed to the level of AV could have been due to S's lailure to retain the low AV trigram during his search. The same type of argument could be applied to the results of the Shulz \& Lovelace (1964) study, as well as to those of the study reported here. As Smith \& Egeth (1966) point out, the materials of the high AV list were somewhat different from those of the low AV list. Therefore, it should be expected that field items of the same AV as the target would interfere more with retention of the target item than would field itens of a different AV. If this argument holds true, then the additional time required in the $\mathrm{H}-\mathrm{H}$ and $\mathrm{L}-\mathrm{L}$ conditions could be explained as time used by $S$ to reexamine (reassimilate) the target item during his search. A replication of the Schulz \& Lovelace (1964) study which adds the independent variable of forced assimilation time of the target (before the field is exposed) should separate the effects directly related to AV from those only indirectly related through short-term memory.

In conclusion, the data support the hypothesis that AV has an effect on perceptual search time but only when the target is in a field of a different AV. A study which forces assimilation of the target and evaluates the role of short-term memory may alter this conclusion.

\section{REFERENCES}

GLAZE, J. A. The association value of nonsense syllables. Journal of Genetic Psychology, $1928,35,255-267$.

PORTNOY, S., PORTNOY, M., \& SALZINGER, $K$. Perception as a function of association value with response bias controlled. Journal of Experimental Psychology, 1964, 68, 316-320. SCHULZ, R. W., \& LOVELACE, E. A. Meaningfulness and the associative phase of paired-associate learning: A methodological consideration. Psychonomic Science, 1964, 1, 37-38.

SMITH, E. E., \& EGETH, H. Effects of association value on perceptual search. Journal of Experimental Psychology, 1966, 71, 687-690.

UNDERWOOD, B. J. Degree of learning and the measurement of forgetting. Journal of Verbal Learning \& Verbal Behavior, 1964, 3, 112-129. 